\title{
Prophylactic treatment for delayed chemotherapy-induced nausea and vomiting after non-AC based moderately emetogenic chemotherapy: a systematic review of randomized controlled trials
}

\author{
Maurice J. D. L van der Vorst ${ }^{1,2}$ - Elisabeth C. W. Neefjes ${ }^{1}$. \\ Inge R. H. M. Konings ${ }^{1}$ - Henk M. W. Verheul ${ }^{1}$
}

Received: 4 September 2014 / Accepted: 17 May 2015 /Published online: 4 June 2015

(C) The Author(s) 2015. This article is published with open access at Springerlink.com

\begin{abstract}
Purpose Delayed chemotherapy-induced nausea and vomiting (CINV) remains an important adverse effect of moderately emetogenic chemotherapy not containing anthracyclines and cyclophosphamide (non-AC MEC). In this review, we summarize current literature to update recommendations for delayed CINV prophylaxis after non-AC MEC. Methods We conducted a systematic search in PubMed and conference proceedings from ASCO, ESMO, and MASCC. Included randomized controlled trials (RCTs) aimed to prospectively evaluate the efficacy of two or more antiemetic strategies in the prevention of delayed CINV after the administration of non-AC MEC. At least one of the following endpoints was used: complete response, complete control, no nausea, no vomiting, and/or no use of rescue medication. Results Our search provided 247 publications. Nine met the predefined criteria. Included RCTs reported outcomes on palonosetron, aprepitant, casopitant, netupitant/palonosetron (NEPA), olanzapine, and megestrol acetate.

Conclusions Superiority of palonosetron over first-generation $5-\mathrm{HT}_{3}$ receptor antagonists for the prevention of acute and delayed CINV after non-AC MEC has not been proven. The
\end{abstract}

Electronic supplementary material The online version of this article (doi:10.1007/s00520-015-2778-6) contains supplementary material, which is available to authorized users.

Henk M. W. Verheul

h.verheul@vumc.nl

1 Department of Medical Oncology, VU University Medical Center, De Boelelaan 1117, 1081 HV Amsterdam, The Netherlands

2 Department of Internal Medicine, Rijnstate Hospital, PO Box 9555, 6800 TA Arnhem, The Netherlands addition of an $\mathrm{NK}_{1}$ receptor antagonist to first-generation 5$\mathrm{HT}_{3}$ receptor antagonists does not significantly improve the incidence of delayed CINV after non-AC MEC. The efficacy of a single-day regimen of dexamethasone with palonosetron is non-inferior to multiday dexamethasone. NEPA, olanzapine, and megestrol acetate show highly effective complete response $(\mathrm{CR})$ rates.

Keywords Antiemetics · Delayed CINV · Moderately emetogenic chemotherapy

\section{Introduction}

Delayed chemotherapy-induced nausea and vomiting (CINV), defined as nausea and vomiting occurring more than $24 \mathrm{~h}$ after completion of chemotherapy, remains an important and common adverse event complicating cancer treatment. Delayed CINV significantly interferes with patient's quality of life (QOL) and daily functioning [1, 2]. Incidence and severity of CINV are affected by patient- and treatment-related factors. Characteristics associated with a higher risk include female sex, anxiety, and poor control with previous chemotherapy $[3,4]$. Delayed CINV is influenced by the effectiveness of control of the acute phase of CINV, as well as the intrinsic emetogenicity of the drug. The risk of delayed CINV has been studied best in chemotherapy regimens containing high-dose cisplatin or anthracyclines and cyclophosphamide (AC) combinations. However, delayed CINV is also associated with moderately emetogenic chemotherapy (non-AC $\mathrm{MEC}$ ) regimens [5-10]. Non-AC MEC consists of a broad range of chemotherapeutic agents, with emetogenic potentials of 30 to $90 \%$; agents like oxaliplatin and irinotecan have an 
emetogenic potential in the lower part of this range, as opposed to carboplatin, which is at the high end $[11,12]$.

In 2013, the Multinational Association for Supportive Care in Cancer (MASCC) and the European Society of Medical Oncology (ESMO) last updated their guidelines for the management of CINV in adults [13]. In 2011, the American Society of Clinical Oncology (ASCO) published the last updated clinical practice guideline for antiemetics in oncology [14]. All guidelines recommend palonosetron combined with dexamethasone for the prevention of acute CINV following non-AC MEC, with multiday oral dexamethasone as the preferred treatment for the prevention of delayed CINV (level of evidence IIb). These recommendations are based however on phase III trials, which did not evaluate the combination of palonosetron and dexamethasone in MEC, but only in highly emetogenic chemotherapy (HEC) and AC chemotherapy $[15,16]$. Also, patients in the aforementioned trials did not receive optimal antiemetic treatment with $\mathrm{NK}_{1}$ receptor antagonists, as recommended for HEC and AC chemotherapy, which may have influenced the results.

Despite the recommended combination of palonosetron and multiday dexamethasone, many patients still experience delayed CINV following non-AC MEC. In a small observational trial with colorectal cancer patients, palonosetron and dexamethasone failed to provide both complete response (CR) and complete control (CC) in $15 \%$ of the patients in the delayed phase [17]. In another phase III trial with palonosetron and dexamethasone administered for 3 days, almost $25 \%$ of the patients did not achieve CR in the delayed phase [18]. Further evidence on the efficacy of new strategies to prevent delayed CINV after non-AC MEC is therefore needed. This systematic review aims to provide a comprehensive assessment of recently performed randomized controlled trials (RCTs) on this specific topic.

\section{Methods}

\section{Search strategy}

We conducted a systematic search in PubMed and conference proceedings of ASCO, ESMO, and MASCC in August 2014. For PubMed, the following syntax was applied: "chemotherapy-induced nausea and vomiting [tiab]" OR "CINV [tiab]" OR "emesis [tiab]" OR "delayed nausea [tiab]" OR "moderately emetogenic [tiab]" OR "MEC [tiab]", with limits: clinical trial, full text, English, humans, adult, and date from January 1 st 2009 to July 31st 2014. An electronic search was undertaken of conference proceedings of ASCO, ESMO, and MASCC from January 1st 2009 to July 31st 2014.

\section{Selection criteria}

Potentially relevant studies retrieved by the PubMed and conference proceedings searches were independently reviewed for eligibility by two investigators (M.V.D.V. and E.C.W.N.). Any disagreement between the reviewers was resolved by reexamination and subsequent discussion to reach a consensus. Unpublished or retrospective studies were not considered eligible. Levels of evidence were not used to assess the value of each publication selected for inclusion. The following criteria for inclusion were applied: (a) The study aimed to prospectively evaluate the efficacy of two or more antiemetic strategies in the prevention of delayed CINV after the administration of moderately emetogenic chemotherapy not containing anthracyclines and cyclophosphamide (non-AC MEC). (b) At least one of the following endpoints was used in the study: (1) complete response, (2) complete control, (3) no nausea, (4) no vomiting, or (5) no use of rescue medication. Studies on patients receiving chemotherapy with mixed emetogenicity (HEC and/or AC and/or non-AC MEC) were included only if subgroup analysis (pre-planned or ad hoc) of the non-AC MEC subgroup was performed. When data on AC and nonAC MEC were combined, it was arbitrarily decided to include studies in which the percentage of patients receiving AC was less than $50 \%$ in the subgroup analysis. Both RCTs in which the antiemetic therapies only differed beyond day 1 , and RCTs in which there was a difference starting at day 1 were included.

\section{Data extraction}

Extracted items were study design, number of patients included, number of patients receiving non-AC MEC, tumor types, emetogenic level of assessed chemotherapeutic agents, primary efficacy endpoints, intervention, and reported results. Because of the heterogeneity in study designs, risk of bias, and variety in patient populations, conducting a meta-analysis was not possible.

\section{Results}

\section{Number of studies meeting selection criteria}

Using the PubMed syntax and aforementioned limits, 247 potentially relevant studies were identified. Figure S1 (available at the Journal of Supportive Care in Cancer online) depicts the subsequent stepwise selection of nine eligible studies. Searching conference proceedings of ASCO, ESMO, and MASCC did not provide additional eligible studies. 


\section{Characteristics eligible studies}

Our search provided nine studies which met the selection criteria [18-26]. Mean sample size was 320 patients. Mean number of patients receiving non-AC MEC was 230. All but three studies assess patients with various tumor types. Most studies assessed antiemetics in patients receiving a broad range of chemotherapy regimens, including HEC, AC, and MEC regimens; three studies assessed non-AC MEC only. Most studies were double-blinded, including one phase II trial. Three had a crossover study design. Two were noninferiority trials. Palonosetron was assessed in three studies, including one evaluating a dexamethasone-sparing regimen. $\mathrm{NK}_{1}$ receptor antagonists were assessed in three studies, including one evaluating casopitant. Although casopitant was discontinued for further development, we included data from this RCT because they provide evidence to clarify the usage of $\mathrm{NK}_{1}$ receptor antagonists for non-AC MEC. Other antiemetics assessed were netupitant/palonosetron (NEPA), olanzapine, and megestrol acetate, each in one study. CR (defined as "no vomiting," and/or "no use of rescue antiemetics") in the acute, delayed, and overall phases was the primary efficacy endpoint in the majority of the studies included. Study details are summarized in Table 1.

\section{Palonosetron}

An open-label, crossover trial was designed to evaluate the efficacy of palonosetron compared with ondansetron [19]. This study included 30 patients with head and neck cancer. All patients received the same non-AC MEC regimen docetaxel $60 \mathrm{mg} / \mathrm{m}^{2}$, carboplatin $300 \mathrm{mg} / \mathrm{m}^{2}$, and 5 -fluorouracil $600 \mathrm{mg} / \mathrm{m}^{2}$. Previous exposure to chemotherapy is unknown. Corticosteroids were administered to all patients on day 1 . CR (defined as no vomiting) and intensity of nausea in the acute, delayed, and overall phases were the primary efficacy endpoints in this study. There were no significant differences in CR rates in all phases (palonosetron vs. ondansetron: acute phase 83.3 vs. $80 \%$; delayed phase 76.6 vs. $66.7 \%$; overall phase 66.7 vs. $46.7 \%$; $p$ values not provided). Differences in control of nausea were also not statistically significant during all phases.

In a multicenter, double-blind, non-inferiority, crossover trial, 144 patients with a broad range of tumor types receiving HEC (cisplatin), AC, or non-AC MEC were randomized to palonosetron in cycle 1 , and then switched to granisetron in cycle 2 or vice versa [20]. Both chemotherapy naïve and nonnaïve patients were included. Corticosteroids were not allowed. The primary efficacy endpoint in this study was the proportion of patients with CR (no vomiting) during the acute, delayed, and overall phases. One hundred and eight patients $(75 \%)$ received AC or non-AC MEC (63\% of patients in this subgroup). One hundred and twenty-two patients received two cycles of chemotherapy. Data of both cycles were pooled. In the mixed $\mathrm{AC} /$ non-AC subgroup, differences in $\mathrm{CR}$ rates between palonosetron and granisetron were not significant; in the acute phase 72.16 vs. $67.65 \%$; in the delayed phase 67.01 vs. $59.80 \%$; in the overall phase 58.76 vs. $52.9 \%$, respectively. $p$ values not provided.

\section{Dexamethasone-reducing study}

A multicenter, open-label, non-inferiority study published in 2011 was designed to evaluate the efficacy of palonosetron plus single-day dexamethasone compared with multiday dexamethasone. This study included 332 patients receiving $\mathrm{AC}$ or non-AC MEC, mainly oxaliplatin-, irinotecan-, and carboplatin-based regimens [18]. All patients were chemotherapy naïve. CR (defined as no vomiting, no use of rescue medication) during the overall phase was the primary outcome measure. In the overall population, differences in $\mathrm{CR}$ rates during the overall phase were significant $(67.5 \%$ for singleday dexamethasone and $71.1 \%$ for dexamethasone on days $1-3$; difference, $95 \% \mathrm{CI}-3.6 \%(-13.5$ to 6.3$))$. In the nonAC MEC subgroup analysis, there were no significant differences in CR rates between the single-day and multiday dexamethasone groups (in the acute phase, 88.3 vs. $87.0 \%$, respectively (difference, $95 \%$ CI 1.3 (-7.6 to 10.2)); in the delayed phase, 71.2 vs. $76.0 \%$, respectively (difference, $95 \% \mathrm{CI}-4.8$ $(-16.7$ to 7$)))$.

\section{$\mathrm{NK}_{1}$ receptor antagonists}

In a multicenter, double-blind study by Rapoport et al., 848 patients receiving $\mathrm{AC}$ and non-AC MEC for a broad range of tumors were randomized to compare the efficacy of an oral three-drug regimen of aprepitant, ondansetron, and dexamethasone to an oral control regimen of ondansetron and dexamethasone [21]. Fifty-two percent of the patients were given non-AC-based MEC, including oxaliplatin, carboplatin, ifosfamide, and irinotecan. All patients were chemotherapy naïve. The primary efficacy endpoint of the study was the proportion of patients reporting no vomiting during the overall phase. In the overall population, a significantly higher proportion of patients on aprepitant reported no vomiting in the overall phase compared to the control group (76.2 vs. $62.1 \%$, respectively, $p<0.001)$. In the post hoc analysis of the nonAC MEC subgroup, statistically more patients in the aprepitant group compared to the control group reported no vomiting; in the acute phase, 96.5 vs. $91.6 \%$, respectively, $p<0.05$; in the delayed phase, 84.5 vs. $73.9 \%$, respectively, $p<0.05$; in the overall phase, 83.2 vs. $71.3 \%$, respectively, $p<0.05$.

In a double-blind, parallel group study by Hesketh et al., enrolling 707 patients receiving non-AC MEC (oxaliplatin) for colorectal cancer, the efficacy of single-dose casopitant 


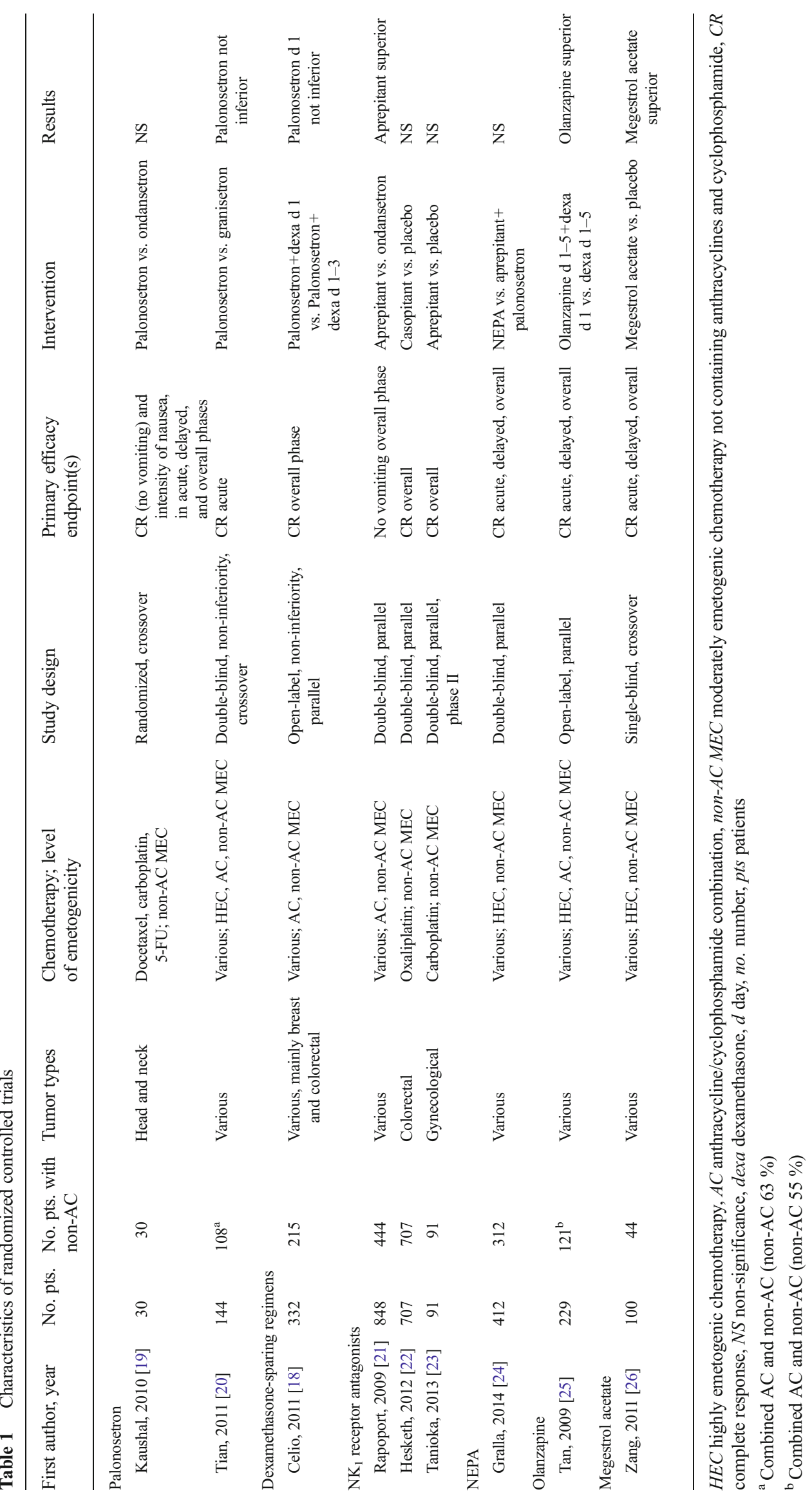


was compared to placebo. All patients received ondansetron on days 1-3 plus dexamethasone on day 1 and were chemotherapy naïve [22]. The primary endpoint in this study was the percentage of patients achieving CR (defined as no vomiting, no use of rescue medication) during the overall phase. There were no significant differences between both groups; $86 \%$ of the patients in the casopitant group vs. $85 \%$ in the placebo group achieved CR $(p=0.7273)$. There were also no significant differences between the casopitant and placebo group in the acute phase ( 97 vs. $96 \%$, respectively, $p=0.4771$ ) and in the delayed phase ( 86 vs. $85 \%$, respectively, $p=0.7273$ ). There was also no significant difference in severity of nausea observed between casopitant and placebo in all phases.

Ninety-one female patients who were younger than 70 years, and received carboplatin-based chemotherapy for gynecological tumors, were randomized to aprepitant or placebo in a multicenter, double-blind, phase II trial [23]. All patients received granisetron and multiday corticosteroids. Previous exposure to chemotherapy is unknown. The primary endpoint in the study was CR (no vomiting, no rescue medication) during the overall phase. CR rates were not significantly different between aprepitant and placebo in the overall phase ( 62 vs. $52 \%$, respectively, $p=0.33$ ). There were also no significant differences in CR rates during the acute and delayed phases between aprepitant and placebo (98 vs. $96 \%$, respectively, and 62 vs. $52 \%$, respectively).

\section{NEPA, a fixed-dose combination of netupitant and palonosetron}

In a multicenter, double-blind study by Gralla et al., the efficacy of a single dose of NEPA (oral fixed-dose combination of $300 \mathrm{mg}$ netupitant and $0.50 \mathrm{mg}$ palonosetron) was compared to oral aprepitant plus oral palonosetron $0.50 \mathrm{mg}$, in $412 \mathrm{pa}-$ tients treated with either HEC or non-AC MEC regimens for a broad range of tumors [24]. Seventy-six percent of the patients received non-AC MEC, mainly carboplatin- and oxaliplatinbased chemotherapy. In this study, the dose/schedule of oral dexamethasone was open label and based on the emetogenicity of the chemotherapeutic regimen. All patients were chemotherapy naïve. The study was not only designed primarily to assess the safety of NEPA, but also assessed the efficacy of this antiemetic drug. Overall incidence, type, and frequency of adverse events were comparable between the treatment groups. In the overall population, CR rates (no vomiting, no use of rescue medication) in the overall phase were similar in cycle 1 ( $81 \%$ in the NEPA group vs. $76 \%$ in the control arm). The reported control of nausea was comparable in both groups: 84-92\% across cycles for NEPA and $81-87 \%$ for the control group. For the non-AC MEC subgroup, $\mathrm{CR}$ rates across cycles were also comparable between the treatment groups: $80-93 \%$ in the NEPA group and $82-$ $89 \%$ in the control group.

\section{Olanzapine}

In 229 patients with a broad range of tumors, the efficacy of olanzapine was compared to the $5-\mathrm{HT}_{3}$ receptor antagonist azasetron in an open-label trial by Tan et al. [25]. Patients receiving $\mathrm{HEC}, \mathrm{AC}$, or non-AC MEC were randomized to olanzapine $10 \mathrm{mg}$ days $1-5$ plus azasetron $10 \mathrm{mg}$ i.v. and dexamethasone $10 \mathrm{mg}$ i.v. on day 1 , or to the control group with azasetron $10 \mathrm{mg}$ i.v. on day 1 plus dexamethasone $10 \mathrm{mg}$ i.v. days $1-5$. Both chemotherapy-naïve and non-naïve patients were included. Fifty-six percent of all randomized patients received AC or non-AC MEC. In this mixed subgroup, $55 \%$ of the patients received non-AC MEC, mainly oxaliplatin-based chemotherapy. The primary endpoint in this study was CR (no nausea and vomiting, no use of rescue medication) during the acute, delayed, and overall phases. In the overall population, $\mathrm{CR}$ rates in the acute phase were very high $(>95 \%)$ and did not significantly differ between olanzapine and 5-day dexamethasone in both the HEC and combined AC and non-AC MEC subgroups. In the combined subgroup, CR rates in the delayed phase were $83 \%$ for the olanzapine group vs. $58 \%$ for the control group $(p<0.05)$; in the overall phase, 83 and $56 \%$, respectively $(p<0.05)$.

\section{Megestrol acetate}

One hundred patients with gastrointestinal or lung cancer, who were treated with HEC or non-AC MEC (mainly oxaliplatin- and irinotecan-based chemotherapy), were randomized in a single-blind, crossover trial published in 2011, to receive either oral megestrol acetate $320 \mathrm{mg}$ or placebo [26]. Corticosteroids were not allowed in this study. Information on previous treatment with chemotherapy was not provided. CR rates in the acute, delayed, and overall phases were primary endpoints. In the non-AC MEC subgroup ( $44 \%$ of all patients), CR rates were significantly higher in the megestrol acetate group: in the overall phase, 50 vs. $27.3 \%$, respectively ( $p=0.002)$; in the acute phase, 72.7 vs. $59.1 \%$, respectively $(p=0.146)$; and in the delayed phase, 52.3 vs. $25.0 \%(p=$ $0.000)$, respectively.

\section{Discussion}

This review focuses on recent RCTs assessing prophylactic antiemetic treatment for delayed CINV following non-AC MEC. Results from the included trials show a diversity of antiemetic agents assessed. Because of heterogeneity in chosen endpoints, including populations, chemotherapy regimens, and tumor types, comparison of data from these studies is limited. There are, however, several findings of interest. We identified two trials comparing the efficacy of palonosetron to first-generation $5-\mathrm{HT}_{3}$ receptor antagonists. Both studies 
suggest that palonosetron is equally effective as firstgeneration $5-\mathrm{HT}_{3}$ receptor antagonists for the prevention of acute and delayed CINV after non-AC MEC. This conclusion is consistent with results from recently performed study by Roscoe et al. [27]. They found that in patients treated with chemotherapy with mixed emetogenicity, including non-AC $\mathrm{MEC}$, palonosetron was not more effective than granisetron in rates of average delayed nausea, which was the primary endpoint in this study, when both were combined with single-day dexamethasone and the dopamine $\left(\mathrm{D}_{2}\right)$ receptor antagonist prochlorperazine. Because no non-AC MEC subgroup analysis was performed, this study did not meet selection criteria for inclusion in our review. On the other hand, the conclusions of both studies included in our review are at variance with results from a recently performed systematic review and metaanalysis by Popovic et al. that showed that palonosetron was superior to first-generation $5-\mathrm{HT}_{3}$ receptor antagonists in preventing acute and delayed CINV [28]. Data from AC and non-AC MEC subgroups were pooled; therefore, this metaanalysis does not specifically address the efficacy of palonosetron for delayed CINV prophylaxis following nonAC MEC.

Obviously, the two included studies regarding palonosetron have some limitations. Both have a crossover design, which causes a considerable risk of bias, including the possibility of a "carry over" of treatment effect from one chemotherapy cycle to the next. The investigators have minimized this risk by pooling data from cycles 1 and 2 . Moreover, the total number of included patients on non-AC MEC regimens was only 138 . Also, multiday dexamethasone for the prevention of delayed CINV was not allowed in both trials, which is not consistent with current guideline recommendations. This could have influenced the outcome measures used in these studies. Furthermore, CR in the delayed phase was not a primary but secondary endpoint in the largest study. It is doubtful whether this trial was powered sufficiently to detect a difference in both endpoints. Considering this, we conclude that at present, there is still insufficient data to decide whether palonosetron is the preferred $5-\mathrm{HT}_{3}$ receptor antagonist following non-AC MEC.

As mentioned before, current guidelines recommend the use of multiday dexamethasone to prevent delayed CINV after non-AC MEC. Dexamethasone use is often accompanied by unpleasant side effects. Reduction of dexamethasone exposure, without a decrease in efficacy, could be beneficial for patients. One study we included in our review reported that the efficacy of a single-day regimen of palonosetron and dexamethasone is non-inferior to palonosetron and multiday dexamethasone in the acute, delayed, and overall phases following non-AC MEC. This evidence may be of particular benefit to patients undergoing multiple cycles of therapy when palonosetron is prescribed and where the long-term side effects of dexamethasone can be reduced.
While the major guidelines do not recommend the use of an $\mathrm{NK}_{1}$ receptor antagonist for non-AC MEC, there is some evidence that adding aprepitant may improve control of vomiting. Results from the study by Rapoport et al. show that a significantly higher proportion of patients on aprepitant reported the primary outcome of no vomiting during all phases. This study, however, has some limitations. For example, nonAC MEC subgroup analysis was not predefined. Furthermore, multiday ondansetron was used as an active control arm for delayed CINV prophylaxis, which is not justified anymore by clinical evidence [29]. Data from two other included studies assessing $\mathrm{NK}_{1}$ receptor antagonists are in contrast with the study by Rapoport et al. Both studies were well-designed and assessed the additional effect of $\mathrm{NK}_{1}$ receptor antagonists to first-generation 5- $\mathrm{HT}_{3}$ receptor antagonists in homogeneous patient populations with clearly defined tumor types, treated with oxaliplatin- or carboplatin-based chemotherapy. Adding $\mathrm{NK}_{1}$ receptor antagonists did not improve $\mathrm{CR}$ rates during the acute and delayed phase. Therefore, we conclude that so far no convincing evidence exists indicating benefits from adding $\mathrm{NK}_{1}$ receptor antagonists to standard prophylactic antiemetic treatment following non-AC MEC.

Some phase II studies suggested that adding a $\mathrm{NK}_{1}$ receptor antagonist to palonosetron and dexamethasone causes high $\mathrm{CR}$ rates in patients undergoing HEC or non-AC MEC [30, 31]. CR rates of more than 95 and $85 \%$ could be achieved during the acute and delayed phase, respectively, for patients treated with carboplatin-based chemotherapy. This led to the drug development of NEPA, an oral fixed-dose combination of netupitant, which is a highly selective $\mathrm{NK}_{1}$ receptor antagonist, and palonosetron. We included the study by Gralla et al., which concluded that this combination drug was safe, well tolerated, and highly effective, when compared to oral 3-day aprepitant and palonosetron, and when both treatment groups are combined with dexamethasone [24]. Because this study was designed primarily to assess the safety of NEPA, we believe that future RCTs should be performed to investigate the efficacy of NEPA in clearly predefined AC and non-AC MEC subgroups.

New anti-CINV regimens for non-AC MEC are evolving. Recently, it was noticed that olanzapine, an atypical antipsychotic, combined with a single dose of dexamethasone and palonosetron was highly effective at controlling acute and delayed CINV in patients receiving HEC [32]. Tan et al. found highly significant $\mathrm{CR}$ rates for delayed CINV prophylaxis following non-AC MEC with multiday olanzapine compared to multiday dexamethasone [25]. Consequently, olanzapine could combine reduction of dexamethasone exposure with improved efficacy. This study has some limitations, however, like its open-label design, which could have influenced outcome measures. Moreover, $\mathrm{AC}$ and non-AC regimens were taken together in the subgroup analysis. Future studies in clearly defined non-AC MEC subgroups should assess 
whether the use of olanzapine results in better CR rates for delayed CINV after non-AC MEC.

The antiemetic potential of megestrol acetate was assessed in one trial in a small population, receiving chemotherapy with mixed emetogenicity [26]. The authors reported highly significant $\mathrm{CR}$ rates for non-AC MEC-treated patients, when megestrol acetate was compared to placebo. All patients received granisetron and metoclopramide, but corticosteroids were not allowed. Subgroup analysis for MEC contained both $\mathrm{AC}$ and non-AC regimens, which could influence the results. Megestrol acetate should be compared in future trials with standard antiemetics.

There are some limitations of this review. Data of the included RCTs could not be synthesized because of the heterogeneity of antiemetic regimens, patient populations, and variance of primary outcomes. AC and non-AC MEC were often combined in subgroup analyses, making it hard to draw firm conclusions for non-AC MEC regimens.

We conclude that high-level evidence for optimal prophylaxis of delayed CINV after non-AC MEC is lacking. We believe that further research is essential to improve antiemetic treatment efficacy and outcome while treatment (dexamethasone)-related toxicities are minimized and acceptable.

Grants This update was not supported by grants.

Conflict of interest The authors declared that they have no competing interests.

Open Access This article is distributed under the terms of the Creative Commons Attribution-NonCommercial 4.0 International License (http:// creativecommons.org/licenses/by-nc/4.0/), which permits any noncommercial use, distribution, and reproduction in any medium, provided you give appropriate credit to the original author(s) and the source, provide a link to the Creative Commons license, and indicate if changes were made.

\section{References}

1. Bloechl-Daum B, Deuson RR, Mavros P, Hansen M, Herrstedt J (2006) Delayed nausea and vomiting continue to reduce patients' quality of life after highly and moderately emetogenic chemotherapy despite antiemetic treatment. J Clin Oncol 24(27):4472-4478

2. Cohen L, de Moor CA, Eisenberg P, Ming EE, Hu H (2007) Chemotherapy-induced nausea and vomiting: incidence and impact on patient quality of life at community oncology settings. Support Care Cancer 15(5):497-503

3. Tonato M, Roila F, Del FA, Ballatori E (1996) Methodology of trials with antiemetics. Support Care Cancer 4(4):281-286

4. Roila F, Ruggeri B, Ballatori E, Del FA, Tonato M (2014) Aprepitant versus dexamethasone for preventing chemotherapyinduced delayed emesis in patients with breast cancer: a randomized double-blind study. J Clin Oncol 32(2):101-106
5. Wymenga AN, van der Graaf WT, Wils JA et al (1996) A randomized, double-blind, multicentre study comparing daily 2 and $5 \mathrm{mg}$ of tropisetron for the control of nausea and vomiting induced by low-dose cisplatin- or non-cisplatin-containing chemotherapy. Ann Oncol 7(5):505-510

6. Gralla R, Lichinitser M, Van Der Vegt S et al (2003) Palonosetron improves prevention of chemotherapy-induced nausea and vomiting following moderately emetogenic chemotherapy: results of a double-blind randomized phase III trial comparing single doses of palonosetron with ondansetron. Ann Oncol 14(10):1570-1577

7. Kaizer L, Warr D, Hoskins P et al (1994) Effect of schedule and maintenance on the antiemetic efficacy of ondansetron combined with dexamethasone in acute and delayed nausea and emesis in patients receiving moderately emetogenic chemotherapy: a phase III trial by the National Cancer Institute of Canada Clinical Trials Group. J Clin Oncol 12(5):1050-1057

8. Pater JL, Lofters WS, Zee B et al (1997) The role of the 5-HT3 antagonists ondansetron and dolasetron in the control of delayed onset nausea and vomiting in patients receiving moderately emetogenic chemotherapy. Ann Oncol 8(2):181-185

9. Hesketh PJ, Bosnjak SM, Nikolic V, Rapoport B (2011) Incidence of delayed nausea and vomiting in patients with colorectal cancer receiving irinotecan-based chemotherapy. Support Care Cancer 19(12):2063-2066

10. Hesketh PJ, Sanz-Altamira P, Bushey J, Hesketh AM (2012) Prospective evaluation of the incidence of delayed nausea and vomiting in patients with colorectal cancer receiving oxaliplatinbased chemotherapy. Support Care Cancer 20(5):1043-1047

11. Hesketh PJ (1999) Defining the emetogenicity of cancer chemotherapy regimens: relevance to clinical practice. Oncologist 4(3): 191-196

12. Herrstedt J, Roila F (2009) Chemotherapy-induced nausea and vomiting: ESMO clinical recommendations for prophylaxis. Ann Oncol 20(Suppl 4):156-158

13. MASCC/ESMO Antiemetic Guideline 2013

14. Basch E, Prestrud AA, Hesketh PJ et al (2011) Antiemetics: American Society of Clinical Oncology clinical practice guideline update. J Clin Oncol 29(31):4189-4198

15. Aapro MS, Grunberg SM, Manikhas GM et al (2006) A phase III, double-blind, randomized trial of palonosetron compared with ondansetron in preventing chemotherapy-induced nausea and vomiting following highly emetogenic chemotherapy. Ann Oncol 17(9):1441-1449

16. Saito M, Aogi K, Sekine I et al (2009) Palonosetron plus dexamethasone versus granisetron plus dexamethasone for prevention of nausea and vomiting during chemotherapy: a double-blind, doubledummy, randomised, comparative phase III trial. Lancet Oncol 10(2):115-124

17. Brugnatelli S, Gattoni E, Grasso D, Rossetti F, Perrone T, Danova M (2011) Single-dose palonosetron and dexamethasone in preventing nausea and vomiting induced by moderately emetogenic chemotherapy in breast and colorectal cancer patients. Tumori 97(3):362-366

18. Celio L, Frustaci S, Denaro A et al (2011) Palonosetron in combination with 1-day versus 3-day dexamethasone for prevention of nausea and vomiting following moderately emetogenic chemotherapy: a randomized, multicenter, phase III trial. Support Care Cancer 19(8):1217-1225

19. Kaushal J, Gupta MC, Kaushal V et al (2010) Clinical evaluation of two antiemetic combinations palonosetron dexamethasone versus ondansetron dexamethasone in chemotherapy of head and neck cancer. Singap Med J 51(11):871-875

20. Tian W, Wang Z, Zhou J et al (2011) Randomized, double-blind, crossover study of palonosetron compared with granisetron for the prevention of chemotherapy-induced nausea and vomiting in a Chinese population. Med Oncol 28(1):71-78 
21. Rapoport BL, Jordan K, Boice JA et al (2010) Aprepitant for the prevention of chemotherapy-induced nausea and vomiting associated with a broad range of moderately emetogenic chemotherapies and tumor types: a randomized, double-blind study. Support Care Cancer 18(4):423-431

22. Hesketh PJ, Wright O, Rosati G et al (2012) Single-dose intravenous casopitant in combination with ondansetron and dexamethasone for the prevention of oxaliplatin-induced nausea and vomiting: a multicenter, randomized, double-blind, active-controlled, two arm, parallel group study. Support Care Cancer 20(7):1471-1478

23. Tanioka M, Kitao A, Matsumoto K et al (2013) A randomised, placebo-controlled, double-blind study of aprepitant in nondrinking women younger than 70 years receiving moderately emetogenic chemotherapy. Br J Cancer 109(4):859-865

24. Gralla R, Bosnjak S, Hontsa A, et al (2014) A phase 3 study evaluating the safety and efficacy of NEPA, a fixed-dose combination of netupitant and palonosetron, for prevention of chemotherapyinduced nausea and vomiting over repeated cycles of chemotherapy. Ann Oncol 25(7):1333-1339

25. Tan L, Liu J, Liu X et al (2009) Clinical research of olanzapine for prevention of chemotherapy-induced nausea and vomiting. J Exp Clin Cancer Res 28:131

26. Zang J, Hou M, Gou HF et al (2011) Antiemetic activity of megestrol acetate in patients receiving chemotherapy. Support Care Cancer 19(5):667-673
27. Roscoe JA, Heckler CE, Morrow GR et al (2012) Prevention of delayed nausea: a University of Rochester Cancer Center Community Clinical Oncology Program study of patients receiving chemotherapy. J Clin Oncol 30(27):3389-3395

28. Popovic M, Warr DG, Deangelis C, et al (2014) Efficacy and safety of palonosetron for the prophylaxis of chemotherapy-induced nausea and vomiting (CINV): a systematic review and meta-analysis of randomized controlled trials. Support Care Cancer 22(6):16851697

29. Geling O, Eichler HG (2005) Should 5-hydroxytryptamine-3 receptor antagonists be administered beyond 24 hours after chemotherapy to prevent delayed emesis? Systematic re-evaluation of clinical evidence and drug cost implications. J Clin Oncol 23(6):1289-1294

30. Kitazaki T, Fukuda Y, Fukahori S, et al (2014) Usefulness of antiemetic therapy with aprepitant, palonosetron, and dexamethasone for lung cancer patients on cisplatin-based or carboplatin-based chemotherapy. Support Care Cancer 23(1):185-190

31. Grunberg SM, Dugan M, Muss H et al (2009) Effectiveness of a single-day three-drug regimen of dexamethasone, palonosetron, and aprepitant for the prevention of acute and delayed nausea and vomiting caused by moderately emetogenic chemotherapy. Support Care Cancer 17(5):589-594

32. Navari RM, Gray SE, Kerr AC (2011) Olanzapine versus aprepitant for the prevention of chemotherapy-induced nausea and vomiting: a randomized phase III trial. J Support Oncol 9(5):188-195 